\title{
Electromagnetically induced transparency in alkali atoms integrated on a semiconductor chip
}

\author{
Aaron R. Hawkins \\ hawkins@ee.byu.edu \\ Holger Schmidt
}

Follow this and additional works at: https://scholarsarchive.byu.edu/facpub

Part of the Electrical and Computer Engineering Commons

\section{Original Publication Citation}

Schmidt, H. and A. R. Hawkins. "Electromagnetically induced transparency in alkali atoms integrated on a semiconductor chip." Applied Physics Letters 86 (25)

\section{BYU ScholarsArchive Citation}

Hawkins, Aaron R. and Schmidt, Holger, "Electromagnetically induced transparency in alkali atoms integrated on a semiconductor chip" (2005). Faculty Publications. 398.

https://scholarsarchive.byu.edu/facpub/398 


\title{
Electromagnetically induced transparency in alkali atoms integrated on a semiconductor chip
}

\author{
H. Schmidta) \\ School of Engineering, University of California Santa Cruz, 1156 High Street, Santa Cruz, \\ California 95064 \\ A. R. Hawkins \\ ECE Department, Brigham Young University, 459 Clyde Building, Provo, Utah 84602
}

(Received 30 August 2004; accepted 23 November 2004; published online 10 January 2005)

\begin{abstract}
We analyze the integration of electromagnetically induced transparency in alkali atoms on a semiconductor chip. We propose the use of antiresonant reflecting optical waveguides with hollow cores to realize the necessary waveguiding capability and optical mode areas of a few square micrometers. We derive the scaling laws and limitations for the reduction in group velocity and single-photon phase shifts as a function of waveguide dimension. We show that miniaturization leads to a large enhancement of single-photon cross-phase modulation, despite the presence of additional limitations due to coherence dephasing and waveguide loss. (C) 2005 American Institute of Physics. [DOI: 10.1063/1.1853528]
\end{abstract}

Electromagnetically induced transparency (EIT) is an optical quantum interference effect that leads to dramatic changes in the optical properties of a medium. ${ }^{1}$ These effects are typically observed in the three-level $\Lambda$-scheme shown in Fig. 1. Application of a probe (Rabi frequency $\Omega_{P}$ ) and a strong coherent coupling field $\left(\Omega_{C}\right)$ between levels $|1\rangle,|2\rangle$, and $|3\rangle$ as shown creates a dark state ${ }^{1}$ and results in linear quantum interference effects (transparency of an otherwise opaque medium, ${ }^{1}$ lasing without population inversion, ${ }^{1}$ and slow and stopped light ${ }^{2,3}$ ). Nonlinear effects such as fourwave mixing, ${ }^{1}$ and sum-frequency generation ${ }^{4}$ can be significantly increased by the quantum interference. Figure 1 shows the specific example of cross-phase modulation. ${ }^{5}$ The application of an additional signal field $\left(\Omega_{S}\right)$ on the $|2\rangle-|4\rangle$ transition leads to enhanced Kerr nonlinearities that result in strong and observable interaction between single photons. ${ }^{5}$

Harnessing the magnitude of EIT effects in an integrated platform has obvious advantages that include robustness, easy alignment, and the possibility of multiple cells on the same device. Various approaches towards integrated EIT have been taken. Induced transparency in III-V semiconductors was demonstrated, ${ }^{6}$ but large coherence dephasing rates led to small effects. Results in doped crystals are impressive; ${ }^{7}$ however, these materials are not suitable for integrated optical devices. Another approach is, therefore, to build alkali vapor cells on a chip in order to combine the large effects in atomic vapors with the advantages of semiconductor technology. Cesium cells with dimensions on the order of millimeters have recently been fabricated with the goal of utilizing dark-state resonances for atomic clocks. ${ }^{8}$ However, while this approach implements the advantages of integration just described, it still relies on bulk optics and collimated beams. A fully integrated optics approach, on the other hand, would take advantage of optical waveguiding for obtaining small mode areas on the order of a few $\mu \mathrm{m}^{2}$ over macroscopic distances on the order of centimeters. Small mode areas lead to significant enhancement of nonlinear effects that depend on the intensity of the applied fields. ${ }^{5,9}$ As

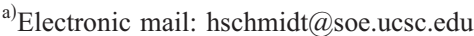

a representative example, the phase shift due to cross modulation in the scheme of Fig. 1 can be expressed as a function of mode area $A$ as ${ }^{5}$

$$
\Phi=\frac{2 \pi N \mu_{13}^{2} \mu_{24}^{2}}{c \varepsilon_{0}^{2} \hbar^{3} \lambda} \frac{P_{S}}{\Omega_{C}^{2} \Delta \omega_{24}} \frac{L}{A},
$$

where $N$ is the atomic density, the $\mu_{i j}$ are the dipole matrix elements of the respective transitions, $P_{S}$ is the power of the signal pulse, and $L$ is the length of the medium. The benefit of maximizing $L$ and minimizing $A$ is evident and provides the motivation for using integrated optics to optimize nonlinear EIT effects at or near room temperature for potential applications.

The purposes of this letter are the following. First, we propose a way of implementing EIT in hot alkali vapor on a semiconductor chip using antiresonant reflecting optical ARROW waveguides with hollow cores. Second, we consider and derive the scaling limits for these integrated devices as a function of the cell (waveguide) dimensions for both linear and nonlinear EIT effects, using cross-phase modulation as an example for nonlinear optics.

Conventional index waveguiding cannot be used to realize integrated EIT because the phase index at the EIT resonance is one. Here, we propose the use of ARROW waveguides with hollow cores for integration of EIT in $\mathrm{Rb}$ atoms. These waveguides work by using highly reflecting

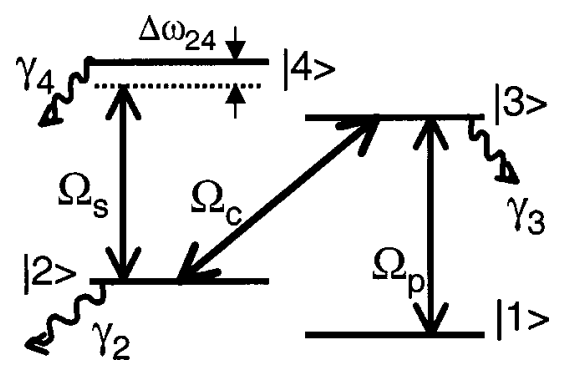

FIG. 1. EIT scheme. Levels $|1\rangle-|3\rangle$ form $\Lambda$-scheme for EIT. A signal field $\left(\Omega_{\mathrm{S}}\right)$ on the $|2\rangle-|4\rangle$ transition induces enhanced cross phase modulation of the probe field $\left(\Omega_{P}\right) . \Omega_{C}$ : coherent coupling field, $\gamma_{i}$ : decay rates. 


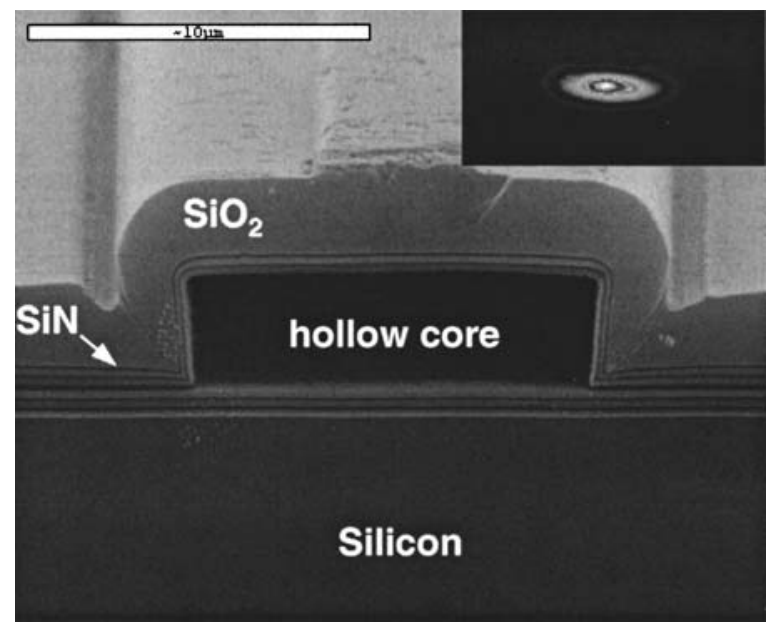

FIG. 2. SEM image of fabricated hollow-core ARROW waveguide after [10] (hollow core: $12 \mu \mathrm{m}$ by $3.5 \mu \mathrm{m}$ ). Inset: Image of observed ARROW mode in air core at output facet.

high-index multilayer claddings around a low index core. Using conventional silicon microfabrication, we have recently demonstrated low-loss propagation at $785 \mathrm{~nm}$ over macroscopic distances in ARROW waveguides with air cores. ${ }^{10}$ A scanning electron microscope (SEM) image of the waveguide is shown in Fig. 2 and the inset shows the observed propagating (single) optical mode with an area (full width at half maximum) of $6.7 \mu \mathrm{m}^{2}$. This is a close match to the atomic absorption cross section of $\mathrm{Rb}: \sigma_{24}=3 \lambda^{2} /(2 \pi)$ $=0.3 \mu \mathrm{m}^{2}$. Losses of a few $\mathrm{cm}^{-1}$ were observed in waveguides up to $1 \mathrm{~cm}$ in length. A vapor cell suitable for EIT measurements can be built by filling and sealing an ARROW waveguide using a similar approach as in Ref. 8 and by coating the walls with paraffin to reduce coherence dephasing (see subsequent discussion). ARROW waveguides with hollow cores represent the first feasible approach to building room-temperature integrated devices based on EIT. They also offer the potential to build multiple EIT cells on one chip and to realize curved beam paths.

Next, we focus on assessing realistic performance limits for such devices, both for linear (slow light) and nonlinear (cross-phase modulation) EIT. Two effects, in particular, need to be considered that distinguish integrated from bulk EIT: the size dependence of the coherence dephasing rate $\gamma_{12}$ and the limiting effect of waveguide losses $\alpha_{\mathrm{WG}}$. For practical operation at room temperature or above and to avoid limitations due to Doppler broadening $\Delta_{\mathrm{D}}$, the dephasing rate $\gamma_{12}$ sets a lower limit for the coupling Rabi frequency via $\Omega_{C}^{2} \geqslant 3 \gamma_{12}\left(\gamma_{3}+\Delta_{\mathrm{D}}\right) . \Omega_{C}$ in turn determines the achievable reduced group velocity ${ }^{2}$ as

$$
v_{g}=\frac{8 \pi}{3 N \lambda^{2}} \frac{\left[\gamma_{12}\left(\gamma_{3}+\Delta_{\mathrm{D}}\right)+\Omega_{C}^{2}\right]^{2}}{\gamma_{r} \Omega_{C}^{2}},
$$

where $\gamma_{r}$ is the radiative decay rate of the atomic transition. To find the size dependence of $\gamma_{12}$, we consider a cylindrical $\mathrm{Rb}$ cell with length $L$, cross section $A$, coated walls, and an inert buffer gas, the latter two of which reduce the probability of dephasing events. The dephasing rate is given by ${ }^{11}$

$$
\alpha_{d}=\frac{\pi N \mu_{13}^{2}}{\varepsilon_{0} \hbar \lambda} \frac{\gamma_{12}}{\Omega_{c}^{2}}
$$




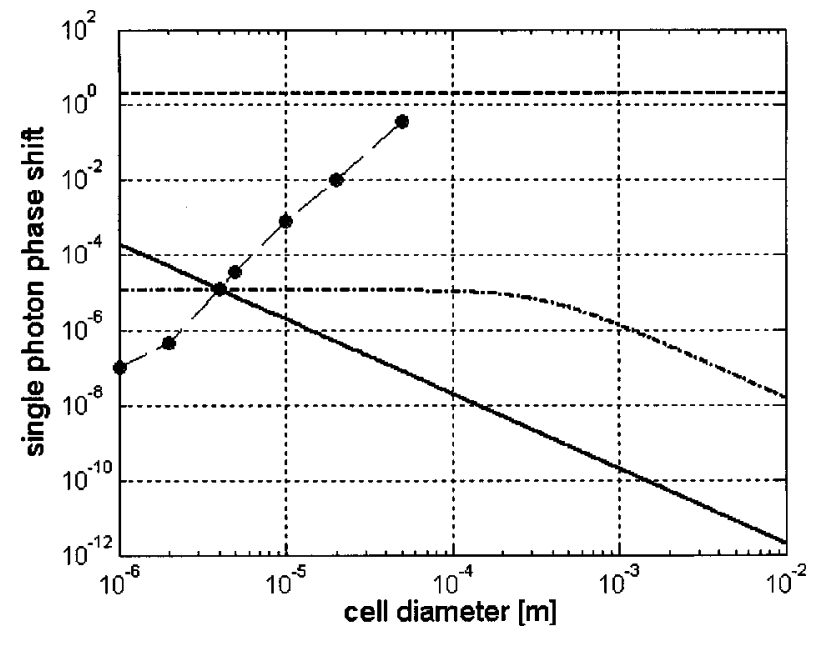

FIG. 4. Limitations of single-photon Kerr phase shift versus EIT cell diameter due to two-photon absorption (dashed line), dephasing-induced absorption (dash-dotted line), waveguide loss (circles), and dispersion (solid line), respectively.

$$
\alpha_{2 P}=\frac{4 \pi^{2} N \mu_{13}^{2} \mu_{24}^{2}}{\varepsilon_{0}^{2} \hbar^{2} \lambda^{2}} \frac{\gamma_{4}}{\Omega_{C}^{2} \Delta \omega_{24} T} \frac{1}{A}
$$

respectively.

The waveguide loss can be calculated from simulations of the ARROW waveguides shown above and increases as the core dimensions are reduced as $\alpha_{\mathrm{WG}} \sim R^{-3} \cdot{ }^{10}$ For all three mechanism, we assume an acceptable absorption-length product of $\alpha_{i} L_{i}=0.1$ to determine $L_{i}$. We then use $L_{i}$ to calculate the single-photon phase shift $\Phi_{1}$ in the linear regime using Eq. (1). Figure 4 summarizes the resulting attainable single-photon phase shift $\Phi_{1}$ in the presence of these limiting mechanisms. Each line represents the attainable phase shift if only one of these mechanisms is considered. The main result is that the dispersion-limited phase shift, which depends inversely on cell area, provides the overall limit down to core dimensions of a few $\mu \mathrm{m}$. This indicates that integration of EIT can indeed lead to much larger nonlinear EIT effects than bulk cells. For example, for a $5 \mu \mathrm{m}$ cell diameter a phase shift of $10^{-5}$ is feasible, which is almost six orders of magnitude higher than for a cell using a collimated beam with a few $\mathrm{mm}$ diameter. This value is independent of pulse width and can always be achieved in a cell that is at least as long as the walkoff length. Two-photon absorption is based on the same third-order nonlinearity as $\Phi_{1}$ and displays the same area dependence. Therefore, $\Phi_{1} / \alpha_{2 P}$ is independent of cell dimension, as seen in Fig. 4. Dephasing and waveguide loss become the limiting factors for the smallest cell dimensions. Since the dephasing-induced loss $\alpha_{d} \sim \gamma_{12} / \Omega_{C}^{2}$, it becomes independent of area in the wall-collision limited range. Consequently, the phase shift scales only with signal pulse width as $\Phi_{1} \sim 1 / T$. One main conclusion is, therefore, that for cell sizes where the phase shift appears dephasing limited, the dispersion limit can be reached by using shorter pulses so that the walkoff length is less than $L_{d}$. We remark that the waveguide losses shown here are not a fundamental limit as they can be further reduced by improved design. They do, however, limit the possible improvement over the dispersion limit by using group velocity matching for the signal pulse as suggested by Lukin and Imamoğlu. ${ }^{13}$

In summary, we have analyzed integration of EIT in alkali atoms on a semiconductor chip. The capability to confine the optical modes in hollow-core waveguides with dimensions on the order of $10 \mu \mathrm{m}$ leads to significant larger nonlinear EIT effects than in bulk or millimeter-sized cells. Hollow-core ARROW waveguides compatible with silicon microphotonics can provide the required waveguiding and mode confinement. They are a promising approach to utilizing the power of EIT effects for studying fundamental photon interaction limits and for applications such as light buffering and information storage. ${ }^{14}$

The authors acknowledge fruitful discussions with D. Yin, J. P. Barber, R. J. Ram, and A. Imamoğlu. Parts of this work are supported by an NSF Career Award (H.S.) and the NIH.

${ }^{1}$ S. E. Harris, Phys. Today 50, 36 (1997), and references therein.

${ }^{2}$ M. M. Kash, V. Sautenkov, A. Zibrov, L. Hollberg, G. Welch, M. Lukin, Y. Rostovtsev, and M. O. Scully, Phys. Rev. Lett. 82, 5229 (1999).

${ }^{3}$ D. F. Philips, A. Fleischhauer, A. Mair, R. Walsworth, and M. D. Lukin, Phys. Rev. Lett. 86, 783 (2001).

${ }^{4}$ S. E. Harris, J. E. Field, and A. Imamoglu, Phys. Rev. Lett. 64, 1107 (1990).

${ }^{5}$ H. Schmidt and A. Imamoglu, Opt. Lett. 21, 1936 (1996).

${ }^{6}$ H. Schmidt, K. L. Campman, A. C. Gossard, and A. Imamoglu, Appl. Phys. Lett. 70, 3455 (1997).

${ }^{7}$ A. V. Turukhin, V. Sudarshanam, M. S. Shariar, J. Musser, B. Ham, and P. R. Hemmer, Phys. Rev. Lett. 88, 023602 (2002).

${ }^{8}$ L. Liew, S. Knappe, J. Moreland, H. Robinson, L. Hollberg, and J. Kitching, Appl. Phys. Lett. 84, 2694 (2004).

${ }^{9}$ S. E. Harris and L. V. Hau, Phys. Rev. Lett. 82, 4611 (1999).

${ }^{10}$ D. Yin, J. P. Barber, A. R. Hawkins, and H. Schmidt, Opt. Express 12, 2710 (2004).

${ }^{11}$ J. Vanier, J. F. Simard, and J. S. Boulanger, Phys. Rev. A 9, 1031 (1974).

${ }^{12}$ H. Schmidt and A. Imamoglu, Opt. Lett. 23, 1007 (1998).

${ }^{13}$ M. D. Lukin and A. Imamoglu, Phys. Rev. Lett. 84, 1419 (2000).

${ }^{14}$ M. D. Lukin, Rev. Mod. Phys. 75, 457 (2003). 